\title{
PRODUÇÃO CIENTÍFICA E FORMAÇÃO DE RECURSOS HUMANOS NA ÁREA 21
}

Joana Zambrano Chambrone ${ }^{1}$, Juliana Souza Uzeloto ${ }^{1}$, Mariana Romanholi Palma ${ }^{1}$, Marcelo Conrado de Freitas ${ }^{1}$, Maria Paula Ferreira de Figueiredo ${ }^{1}$, Monique Yndawe Castanho Araujo ${ }^{1}$, Natália Turri da Silva ${ }^{1}$, Rômulo Araújo Fernandes ${ }^{2}$

Universidade Estadual Paulista - UNESP, ${ }^{1}$ Programa de Pós Graduação em Fisioterapia. ${ }^{2}$ Departamento de Educação Física, Presidente Prudente, SP. E-mail: jo_chambrone@hotmail.com

\section{RESUMO}

A universidade é grande responsável pela produção de pesquisa básica e conhecimento, colaborando com o desenvolvimento econômico do país. Em meio a isso, foram delineados os indicadores bibliométricos, responsáveis por análises de custo-efetividade para investimentos em pesquisa. O presente estudo buscou analisar a relação entre concluir, ou não, doutorado na Área 21 e indicadores de produção científica e formação de recursos humanos entre 2010 e 2013, em docentes com bolsa produtividade. Foi realizada busca no portal do CNPq de docentes "Bolsistas Produtividade do CNPq" da área 21 nas classes 1A, 1B, 1C, 1D e 2. Os currículos dos docentes foram verificados por meio da Plataforma Lattes e separados em dois grupos de acordo com o doutoramento: Área 21 e Outros. Pesquisadores com doutoramento na área 21 apresentam 26,7\% dos artigos publicados em periódicos A1, mostrando associação positiva com aumento de 4,485 vezes em relação aos pesquisadores com doutoramento em outras áreas.

Palavras-chave: Indicadores Bibliométricos, educação física e treinamento, fisioterapia, Fonoaudiologia, Terapia Ocupacional.

\section{SCIENTIFIC PRODUCTION AND TRAINING OF HUMAN RESOURCES IN THE AREA 21}

\section{ABSTRACT}

The university is largely responsible for the production of basic knowledge and research, collaborating with the country's economic development. Through it, the bibliometric indicators, responsible for cost-effectiveness analysis for investments in research were outlined. This study investigates the relationship between complete or not, doctorate in Area 21 and indicators of scientific production and training of human resources between 2010 and 2013 in teachers with productivity scholarship. A search was performed in CNPq portal of teachers "Fellows Productivity CNPq" Area 21 in classes 1A, 1B, 1C, 1D and 2. The curriculum of teachers have been verified by the Lattes Platform and separated into two groups according to the doctorate: Area 21 and Other. Researchers with a PhD in the area 21 have $26.7 \%$ of articles published in journals $A 1$, showing a positive association with increased 4,485 times compared to researchers with doctorate in other areas.

Keywords: Bibliometric Indicators, Physical Education and Training, Physical Therapy Specialty, Speech, Language and Hearing Sciences, Occupational Therapy. 


\section{INTRODUÇÃO}

As pesquisas em saúde avançaram de forma significativa nos últimos anos em todo o mundo, estando este aumento relacionado ao maior número de pesquisadores e de programas de pós-graduação que passaram a focalizar esta temática ${ }^{1 .}$ Outro fator contribuinte é o maior interesse em prevenção de doenças e qualidade de vida de indivíduos saudáveis ou com doenças crônicas $^{1,2}$, os quais refletiram nos altos investimentos em recursos humanos, atenção básica, ciência e tecnologia nos últimos vinte anos ${ }^{3}$.

As pesquisas estão em grande parte vinculadas à pós-graduação, sendo que o desenho da pós graduação brasileira, estabelecido desde 1960, indica a universidade como a grande responsável pela produção de pesquisa básica e conhecimento, os quais colaboram com o desenvolvimento econômico do país. Em meio a isso foram delineados os indicadores bibliométricos, os quais tornaram-se responsáveis por análises de custo-efetividade para investimentos em pesquisa e com isso a competitividade por fomento incrementou a competição por produtividade em pesquisa ${ }^{4}$.

A avaliação trienal na área 21 - a qual inclui educação física, fisioterapia, fonoaudiologia e terapia ocupacional - dos anos 2007-2009 valorizou bastante o quesito quantitativo de "produção intelectual", sendo este representativo de $40 \%$ da avaliação da pós-graduação, o qual englobou também a produção de livros e capítulos, até então pouco explorados ${ }^{5}$.

A Área 21 teve um bom desempenho, sendo que do total de programas de pós-graduação (PPGs) avaliados, $52 \%$ não alteraram o conceito, $28 \%$ aumentaram e apenas $8 \%$ tiveram conceitos diminuídos. Ela ainda contava com 35 PPGs até o final do triênio 2004-2006 e no decorrer do triênio 2007-2009 foram aprovados cinco novos, além de dois novos cursos de doutorado criados em PPGs já existentes. Com isso, somam-se 40 PPGs, envolvendo 56 cursos $^{5}$.

Apesar do aumento no número de programas de pós-graduação na área 21 , muitos profissionais buscam suas formações em programas de doutorado em outras áreas. Em meio a isso, o presente estudo buscou analisar a relação entre concluir (ou não) o Doutorado na Área 21 e indicadores de produção científica e formação de recursos humanos entre os anos de 2010 e 2013 em docentes com bolsa de produtividade do CNPq, os quais fazem parte da elite acadêmica do país. 


\section{METODOLOGIA}

O presente estudo tem caráter descritivo e exploratório. Para seleção da amostra, em setembro de 2014, foi realizada uma busca no portal do CNPq de docentes vinculados à área 21 e que fossem "Bolsistas Produtividade do CNPq", de acordo com as classes 1A,1B, 1C, 1D e 2.

Após identificação desses "Bolsistas Produtividade", por meio da Plataforma Lattes do CNPq, três pesquisadores consultaram os currículos dos docentes. A plataforma Lattes é utilizada nacionalmente e também internacionalmente, como uma forma de registro da produção científica e da carreira acadêmica de pesquisadores. Ela funciona como um registro aberto de informações e detalhes, além de ter mecanismos que permitem uma maior confiabilidade e integridade das informações declaradas (Conselho Nacional de Desenvolvimento Científico e Tecnológico - CNPq. Histórico. 2012) ${ }^{6}$.

Sequencialmente foi construído um banco de dados (no programa Excel, versão 2007) com informações do período de 2010 a 2013, referentes à: i) formação acadêmica, ii) produção científica, iii) participação em eventos, iiii) formação de recursos humanos. Um quarto pesquisador conferiu os dados computados para maior fidedignidade no processamento, e novamente a planilha foi conferida pelos 3 pesquisadores anteriores, caracterizando uma dupla checagem.

Os docentes foram separados em dois grupos de acordo com o doutoramento: Área 21 (doutorado concluído na área 21) e Outros (doutorado concluído em outra área) para verificarmos a relação da área de conclusão do doutorado com indicadores de produção científica e formação de recursos humanos. Os indicadores de produção científica constituem-se por: número total de artigos publicados, número total de artigos publicados em revistas de qualis $\mathrm{A} 1$, número de livros e capítulos de livro. Para formação de recursos humanos foram somadas as orientações e coorientações em mestrado e doutorado já concluídas no período referido.

\section{Análise estatística}

Para análise dos dados foi utilizado o programa estatístico SPSS versão 15.0. Para analisar a normalidade dos dados foi utilizado o teste de Shapiro-Wilk. Para comparação entre os grupos foi realizado o teste $t$ independente para dados de distribuição normal (total de artigos) e o teste de Mann-Whitney para dados com distribuição não normal (demais dados). Para análise de variáveis ao longo do tempo, foi realizado o teste de ANOVA para medidas repetidas ajustado pelo co-fator área 21. Também foi realizado o teste de Qui-Quadrado para avaliar a presença de associação, e quando essa foi verificada, realizamos a regressão logística de Odds Ratio. 


\section{RESULTADOS}

Dos 85 "Bolsistas Produtividade do CNPq" encontrados, quatro pesquisadores estão alocados na classificação $1 \mathrm{~A}$, dez em 1B, cinco em 1C, quinze em $1 \mathrm{D}$ e cinquenta e um na classificação 2 . Observou-se que $38,82 \%$ são do sexo masculino e $61,18 \%$ do feminino, apenas 43,53\% possuem pós-doutorado sendo que destes, 41,18\% concluíram antes do período de 2010. Um total de 45 bolsistas concluiu o doutorado na área 21. Na tabela 1 estão descritos os valores de caracterização da amostra, produtividade e recursos humanos, separados por área.

Tabela 1. Valores expressos em mediana e valores mínimo e máximo.

\begin{tabular}{cccc}
\hline & Área 21 $(\mathrm{n}=45)$ & Outros $(\mathrm{n}=40)$ & Valor de $p$ \\
\hline$\frac{\text { Caracterização }}{\text { Gênero (M/F) }}$ & & & \\
Pós-doutorado & $16 / 29$ & $16 / 24$ & \\
& $1(0 ; 3)$ & $1(0 ; 2)$ & 0,679
\end{tabular}

\section{$\underline{\text { Produtividade }}$}

$\begin{array}{llll}\text { Total de artigos } & 27(5 ; 67) & 28,50(6 ; 74) & 0,868 \\ \text { Publicações A1 } & 4(0 ; 19) & 2(0 ; 9) & 0,099 \\ \% \text { A1 } & 13,70(0 ; 60) & 9(0 ; 33) & 0,051 \\ \text { Livro } & 0(0 ; 1) & 0(0 ; 3) & 0,984\end{array}$
Capítulo de livro
$1(0 ; 14)$
$1(0 ; 20)$
0,880

Congresso

$7(0 ; 29)$

$6(0 ; 26)$

0,316

$\underline{\text { Recursos humanos }}$
$7(2 ; 19)$
$9(0 ; 23)$
0,155

$M=$ masculino; $F=$ feminino

$(p<0,05)$

Para a análise de associação entre percentual de produção em revista A1 e doutoramento na área 21 foi utilizado o ponto de corte de $25 \%$ para produtividade (Tabela 2). 
Tabela 2. Associação entre percentual de produtividade A1 e doutorado na área 21.

\begin{tabular}{|c|c|c|c|c|}
\hline & \multicolumn{4}{|l|}{ Percentual de A1 } \\
\hline & Menor que $25 \%$ & Maior que $25 \%$ & Total & Valor de $p$ \\
\hline Área 21 & $33(73,3 \%)$ & $12(26,7 \%)$ & 45 & \\
\hline Outros & $37(92,5 \%)$ & $3(7,5 \%)$ & 40 & $0,024 *$ \\
\hline Total & $70(82,4 \%)$ & $15(17,6 \%)$ & 85 & \\
\hline
\end{tabular}

* Teste de Qui-Quadrado com nível de significância menor que 0,05 $(p<0,05)$.

Utilizando-se o modelo de regressão logística, foi identificado (Tabela 2) que pesquisadores com doutoramento na área 21 possuem mais chances de publicar em revista estrato A1 quando comparados com pesquisadores com doutoramento em outras áreas, sendo demonstrado o valor de Odds Ratio $(4,485)$, intervalo de confiança $(1,163$ - 17,289) e significância estatística $(p=0,029)$.

Segundo a produtividade ao longo do tempo, para os anos de 2010 a 2013, observou-se um crescimento em ambos os grupos, tanto para os pesquisadores com doutorado na área 21 como para outras áreas. As curvas mantiveram um padrão similar, apresentando produção crescente em 2013, com diferença significante quando comparados com 2010, 2011 e 2012. Não houve diferenças entre os grupos para nenhum dos momentos (Figura 1).

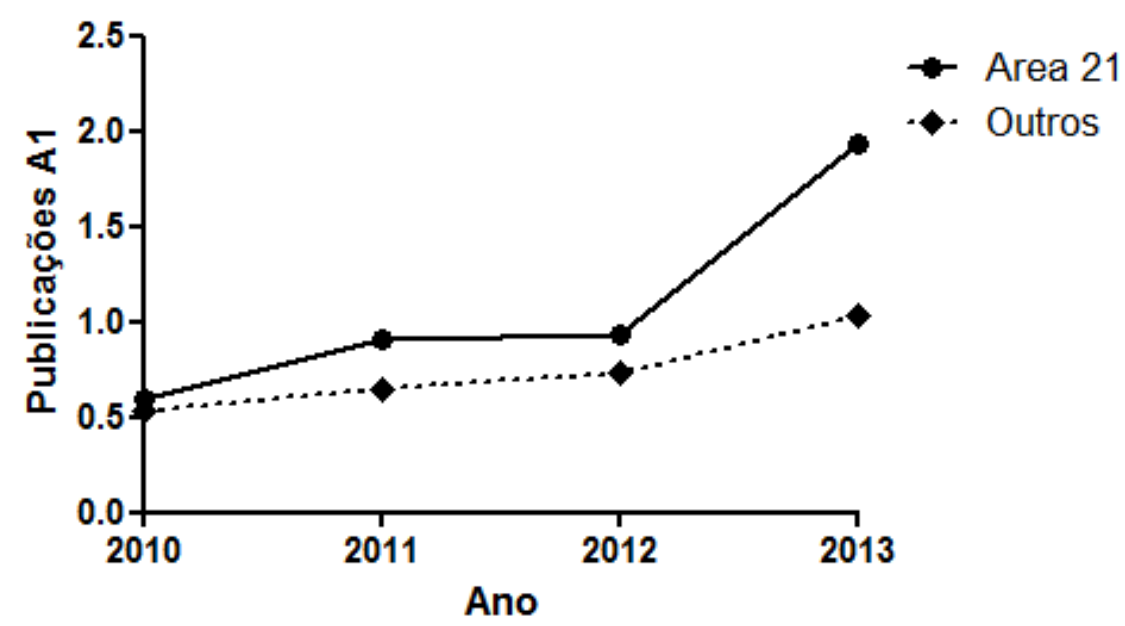

Figura 1. Análise de publicações A1 ao longo do tempo, utilizou-se o teste de ANOVA para medidas repetidas ajustado pelo co-fator área 21. 


\section{DISCUSSÃO}

O presente estudo buscou analisar a relação entre concluir (ou não) o Doutorado na Área 21 e indicadores de produção científica e formação de recursos humanos em docentes com bolsa de produtividade do $\mathrm{CNPq}$,

Em uma análise primária buscou-se uma visão geral da população pesquisadora $P Q$ da área 21, e pode-se observar maior prevalência de mulheres. Tal fato pode estar relacionado com o processo histórico da profissão de fisioterapia no Brasil a qual contempla maior número de mulheres trabalhando tanto em pesquisa quanto em ensino nos cursos de Fisioterapia ${ }^{7,8}$.

Em relação aos pesquisadores $P Q$ em um contexto geral, sabe-se que estes possuem papel importante na formação de recursos humanos qualificados. No presente estudo buscou-se separar a área 21 de outras áreas de doutoramento e pode-se notar que profissionais que concluíram seu doutorado em outra área, que não a 21, apresentaram um maior número de orientações/coorientações de mestrados e doutorados, embora esta diferença não tenha se mostrado estatisticamente significante.

Em relação a isso, a recente regulamentação da fisioterapia no Brasil pode ter contribuído com o baixo nível de doutoramento na área, haja visto o primeiro programa de pesquisa de pósgraduação nessa área, o qual tem menos de $20 \operatorname{anos}^{8}$. Além disso, a Educação Física, parceira da área, contava com dois programas de mestrado e nenhum de doutorado em 1980 sendo que em 2000 esses números subiram para doze e em 2010 a área contava com vinte e um cursos de mestrado e nove de doutorado. Tais dados demonstram o significativo avanço na área da saúde nos últimos anos 9 .

Esse desenvolvimento da área 21 também pôde ser visualizado ao decorrer dos anos, com um crescimento significativo de publicações, em periódicos A1, do ano de 2010 para 2013. Apesar de outras áreas apresentarem um crescimento de publicações relevantes contínuas, isso mostra que essa área não está estacionada, mas sim em desenvolvimento. Dados apontam que a área 21 cresceu em 22\%, pois eram 41 Programas de Pós-graduação (PPGs) no triênio de 2007-2009 passando para 51 no triênio de 2010-2012, além disso, este crescimento envolve também termos qualitativos, com aumento no número de cursos com notas elevadas ${ }^{10}$.

O presente estudo demonstrou que não há diferença entre os grupos em relação a publicações totais de artigos, no entanto, os pesquisadores com doutoramento na área 21 apresentam $26,7 \%$ dos artigos publicados em periódicos A1, mostrando associação positiva com aumento de 4,485 vezes em relação aos pesquisadores com doutoramento em outras áreas. 
Se, por um lado, este panorama de destaque da produção intelectual ganha força neste processo da avaliação da pós-graduação stricto sensu, por outro é preocupante o reduzido número de periódicos internacionais especializados e de forte impacto nesta área, especialmente no processo de composição de um Qualis Periódico que atenda satisfatória e integralmente as quatro áreas profissionais que a compõem (Educação Física, Fisioterapia, Fonoaudiologia e Terapia Ocupacional) ${ }^{5}$.

Embora o contexto da baixa qualidade ainda persista de uma maneira geral, ou seja, ainda não se pode considerar a área 21 como área "ouro" o resultado deste estudo revelou que apesar dos grupos apresentarem similaridade na produtividade total, os pesquisadores com doutoramento na área 21 atingem publicações de maior qualidade.

Este fato pode ser explicado por maiores investimentos na área 21, como aumento do número de PPGs nos últimos 3 anos ${ }^{10}$, que desta maneira atraem investimentos e motivam os pesquisadores a melhorar o conceito, uma vez que a quantidade de cursos stricto sensu com conceitos seis e sete avaliados pela CAPES, mostram correlação de 0,75 , indicando que quanto maior o investimento nas universidades mais cursos bem qualificados ela terá ${ }^{11}$.

Também a linha de pesquisa adotada pode ser fator contribuinte, uma vez que os pesquisadores geralmente seguem estudos similares aos desenvolvidos em sua formação acadêmica, tendo maior engajamento nesta área.

\section{CONCLUSÃO}

Pesquisadores com doutoramento na área 21 possuem mais publicações em revistas de estrato de qualificação A1 quando comparados aos pesquisadores com doutoramento em outras áreas, porém não diferem quanto à formação de recursos humanos.

\section{REFERÊNCIAS}

1. Barros MVG. A pesquisa e formação de pesquisadores em atividade física e saúde no Brasil. Rev. bras. ativ. fís. saúde 2012 Jun; 17(3):161-2.

2. Turato ER. Métodos qualitativos e quantitativos na área da saúde: definições, diferenças e seus objetos de pesquisa. Ver Saúde Publica. 2005; 39(3):507-514. http://dx.doi.org/10.1590/S0034$\underline{89102005000300025}$

3. Paim J, Travassos C, Almeida C, Bahia L, Macinko J. Saúde no Brasil 1: O sistema de saúde brasileiro: história, avanços e desafios. Lancet 2011 Maio;377(9779): 11-31. 
4. Vitor-Costa $M$, Maia-da-Silva $P$, Soriano JB. A avaliação da produtividade em pesquisa na Educação Física: reflexões sobre algumas limitações dos indicadores bibliométricos. Rev. bras. educ. fís. esporte 2012Dez; 26(4): 581-597.

5. Costa D. Avaliação Trienal 2007-2009 dos Programas de Pós-graduação da Área 21 pela CAPES. Revbrasfisioter. [online]. 2010 Dez;14(6): v-vii.

6. (Conselho Nacional de Desenvolvimento Científico e Tecnológico - CNPq. Histórico. 2012).

7. Machado MH, Wermelinger M, Tavares MFL, Moysés NMN, Teixeira M, Oliveira ES. Análise da força de trabalho do setor saúde no Brasil: focalizando a feminização. Rio de Janeiro: Fundação Oswaldo Cruz, Rede Observatório de Recursos Humanos em Saúde; 2006.

8. Sturmer G, Viero CCM, Silveira MN, Lukrafka JL, Plentz RDM. Profile and scientific output analysis of physical therapy researchers with research productivity fellowship from the Brazilian National Council for Scientific and Technological Development. Braz J PhysTher. 2013Feb; 17(1):41-8. http://dx.doi.org/10.1590/s1413-35552012005000068

9. Rigo LC, Ribeiro GM, Hallal PC. Unidade na diversidade: desafios para a Educação Física no século XXI. RevBrasAtivFis e Saúde 2011Ago; 16(4):339-45.

10.Rodacki, ALF.,lorio, M. C. M., \& de Jesus Guirro, R. R. A avaliação trienal dos programas de pósgraduação da Área 21. Fisioter. Pesqui. 2013Dec; 20(4), 307.

11. Soares TC, Serra FAR, Mazon G, Herling LHD. Correlação entre valor repassado pelo estado e conceito capes nas universidades federais brasileiras. IX Colóquio Internacional Sobre Gestão Universitária na América do Sul, Mar del Plata 2009. 